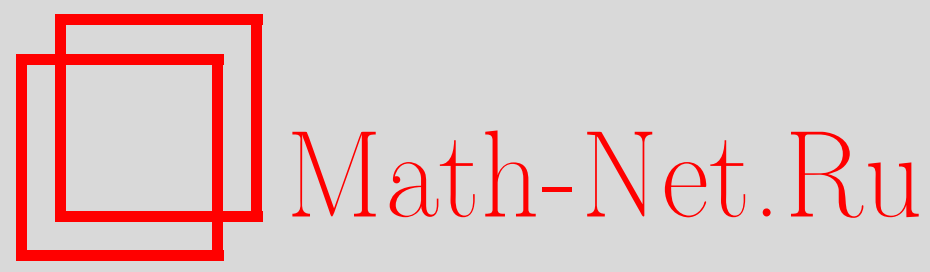

P. K. Ахунжанов, О распределении по модулю 1 экспоненциальных последовательностей, Матем. заметки, 2004, том 76, выпуск 2, 163-171

DOI: https://doi.org/10.4213/mzm96

Использование Общероссийского математического портала Math-Net.Ru подразумевает, что вы прочитали и согласны с пользовательским соглашением http://www.mathnet.ru/rus/agreement

Параметры загрузки:

IP : 54.197 .217 .227

26 апреля 2023 г., 14:07:08 


\section{О РАСПРЕДЕЛЕНИИ ПО МОДУЛЮ 1 ЭКСПОНЕНЦИАЛЬНЫХ ПОСЛЕДОВАТЕЛЬНОСТЕЙ}

\section{Р.К. Ахунжанов}

Получены новые количественные результаты о пересечении выигрышных множеств и его хаусдорфовой размерности. Имеется приложение к задаче о дробных долях $\left\{2^{n} 3^{m} \alpha\right\}$.

Библиография: 10 названий.

1. Введение и формулировки результатов. Пусть $Q=\left\{q_{n} \mid n=1,2,3, \ldots\right\}$ достаточно быстро растущая последовательность положительных действительных чисел, а $\psi(n)$ - некоторая положительнозначная достаточно медленно убывающая к нулю функция. В настоящей работе исследуется множество вещественных чисел $x$ таких, что $\left\|q_{n} x\right\| \geqslant \psi(n)$ при всех натуральных $n$ ( $\|$ · $\|$ означает расстояние до ближайшего целого). Через $\mathrm{HD}(P)$ мы будем обозначать хаусдорфову размерность множества $P$ действительных чисел. Через $\mathrm{ND}(Q)$ обозначим множество таких вещественных $x$, для которых последовательность $\left(q_{n} x\right)_{n=1}^{\infty}$ не всюду плотна.

Эрдеш в работе [1] поставил следующий вопрос: верно ли, что если последовательность натуральных чисел $Q$ такова, что

$$
\forall n \in \mathbb{N} \quad \frac{q_{n+1}}{q_{n}} \geqslant \lambda>1,
$$

то $\mathrm{ND}(Q) \neq \mathbb{Q}$ ? Положительный ответ на этот вопрос был дан де Матаном [2] и Поллингтоном [3]. Они доказали, что если последовательность неотрицательных действительных чисел $Q$ такова, что выполняется $(1)$, то $\operatorname{HD}(\mathrm{ND}(Q))=1$.

Отметим, что Бошерницан в работе [4] доказал, что если

$$
\lim _{n \rightarrow \infty} \frac{q_{n+1}}{q_{n}}=1
$$

то $\mathrm{HD}(\mathrm{ND}(Q))=0$.

Упомянем также работу Фюрстенберга [5] (см. также [6]), в которой для последовательности $Q$, представляющей собой “нелакунарную полугруппу целых чисел по умножению" (например, в качестве такой последовательности можно взять последовательность целых чисел вида $2^{m} 3^{n}$, упорядоченную по возрастанию), доказано, что для иррационального $x$ дробные доли $\left\{q_{n} x\right\}$ всюду плотны (таким образом, для последовательности $Q$, состоящей из чисел $2^{m} 3^{n}$, будет выполнено $\left.\mathrm{ND}(Q)=\mathbb{Q}\right)$.

Работа выполнена при поддержке Российского фонда фундаментальных исследований, грант № 02-01-00912. 
С другой стороны, Шмидт в работе [7], рассматривая множество чисел, не являющихся нормальными ни по какому целому основанию, точнее, множество

$$
T=\left\{x \in \mathbb{R} \mid\left\{q^{n} x\right\}, n \in \mathbb{N}, \text { не всюду плотна для любого целого } q>1\right\},
$$

используя технику вьпгрьшшых множеств, доказал, что $\operatorname{HD}(T)=1$.

В настоящей статье мы обобщим некоторые из этих результатов, а также получим их количественные аналоги.

Нам понадобятся следуюшие обозначения: $\mathbb{N}_{0}$ - множество натуральных чисел с нулем; $\lfloor\cdot\rfloor-$ ближайшее целое снизу; $\bar{\jmath}=\max \{j, 3\}$.

Сформулируем наши результаты.

ТЕОРема 1. Пусть $\left(v_{j}\right)_{j=1}^{\infty}-$ последовательность полохительных действительных чисел таких, что $\sum_{j=1}^{\infty} v_{j}^{-1}=A, u \varkappa \geqslant \varkappa_{0}=\ln \left(2^{18} 3^{2}\right)$. Тогда существует мнохсество $P_{\varkappa}$ вещественных чисел $x$ такое, что

$$
\forall x \in P_{\varkappa} \quad \forall n \in \mathbb{N}_{0} \quad \forall j \in \mathbb{N} \quad \text { выполнена оценка }\left\|2^{n} 3^{j} x\right\| \geqslant \exp \left(-2 A \varkappa v_{j}\right)
$$

$u \operatorname{HD}\left(P_{\varkappa}\right) \geqslant 1-\varkappa_{0} / \varkappa$.

ТЕОРема 2. Пусть $\left(v_{j}\right)_{j=1}^{\infty}-$ последовательность полохсительных действительных чисел таких, что $\sum_{j=1}^{\infty} v_{j}^{-1}=A, u \varkappa \geqslant \varkappa_{0}=\ln \left(2^{18} 3^{2}\right)$. Тогда существует мнохсество $P_{\varkappa}$ вещественных чисел $x$ такое, что

$\forall q \in \mathbb{Z}, q \geqslant 2, \quad \forall x \in P_{\varkappa} \quad \forall n \in \mathbb{N}_{0} \quad$ выполнена оценка $\left\|q^{n} x\right\| \geqslant \exp \left(-2 A \varkappa v_{q}\right)$ $u \operatorname{HD}\left(P_{\varkappa}\right) \geqslant 1-\varkappa_{0} / \varkappa$.

СлЕДСТВИЕ 1. Пусть $\varepsilon>0, \varkappa \geqslant \varkappa_{0}=\ln \left(2^{18} 3^{2}\right) u$

$$
A=\sum_{j=1}^{\infty} \frac{1}{j(\ln \bar{\jmath})^{1+\varepsilon}} .
$$

Тогда существует мнохсество $P_{\varkappa}$ вещественных чисел $x$ такое, что $\forall x \in P_{\varkappa} \quad \forall n \in \mathbb{N}_{0} \quad \forall j \in \mathbb{N} \quad$ выполнена оценка $\quad\left\|2^{n} 3^{j} x\right\| \geqslant \exp \left(-2 A \varkappa j(\ln \bar{j})^{1+\varepsilon}\right)$ $u \operatorname{HD}\left(P_{\varkappa}\right) \geqslant 1-\varkappa_{0} / \varkappa$.

СлЕДСТВИЕ 2. Пусть $\varepsilon>0, \varkappa \geqslant \varkappa_{0}=\ln \left(2^{18} 3^{2}\right) u$

$$
A=\sum_{j=1}^{\infty} \frac{1}{j(\ln \bar{\jmath})^{1+\varepsilon}} .
$$

Тогда существует мнохсество $P_{\varkappa}$ вещественных чисел $x$ такое, что $\forall q \in \mathbb{Z}, q \geqslant 2, \quad \forall x \in P_{\varkappa} \forall n \in \mathbb{N}_{0} \quad$ выполнена оценка $\left\|q^{n} x\right\| \geqslant \exp \left(-2 A \varkappa q(\ln \bar{q})^{1+\varepsilon}\right)$ $u \operatorname{HD}\left(P_{\varkappa}\right) \geqslant 1-\varkappa_{0} / \varkappa$. 
ЗАмЕчаниЕ. Аналогичньй результат, но с более слабыми оценками, был получен в статье [8].

2. О хаусдорфовой размерности. Для оценки снизу хаусдорфовой размерности будет использована следующая теорема.

Теорема 3 (Эгглстон [9]). Пусть $A_{k}=\bigsqcup_{i=1}^{R_{k}} \Delta_{k}(i)$, әде $\Delta_{k}(i)$-интервал длинь $\ell\left(\Delta_{k}(i)\right)=\delta_{k}, k \in \mathbb{N}$. И пусть кажддй интервал $\Delta_{k}(i)$ содержит в точности $N_{k+1}>1$ дизгюнктных интервалов (длины $\left.\delta_{k+1}\right)$ из мноэсества $A_{k+1}$ (при этом $\left.R_{k+1}=R_{k} \cdot N_{k+1}\right)$. Предположим, что $0<s_{0} \leqslant 1$ и что при любом $s<s_{0}$ сходится ряд

$$
\sum_{k=2}^{\infty} \frac{\delta_{k-1}}{\delta_{k}}\left(R_{k}\left(\delta_{k}\right)^{s}\right)^{-1}
$$

Тогда мнохсество $A=\bigcap_{k=1}^{\infty} A_{k}$ имеет хаусдорфову размерность $\operatorname{HD}(A) \geqslant s_{0}$.

3. О выигрышных множествах. В этом пункте мы переформулируем нужным нам образом некоторые результаты В. Шмидта из [7], [10].

Пусть $0<\alpha<1,0<\beta<1$ и $\rho>0$. Пусть на вещественной оси отмечено некоторое множество точек $S \subseteq \mathbb{R}$. Рассматривается игра, в которую играют черные и белые. Сначала черные выбирают на действительной оси замкнутьй отрезок $B_{1}$ длины $\ell\left(B_{1}\right)=2 \rho$. Затем белые выбирают замкнутый отрезок $W_{1} \subset B_{1}$ длины $\ell\left(W_{1}\right)=\alpha \ell\left(B_{1}\right)$. Потом черные выбирают замкнутый отрезок $B_{2} \subset W_{1}$ длины $\ell\left(B_{2}\right)=\beta \ell\left(W_{1}\right)$, и т.д. Таким способом порождается последовательность вложенных замкнутых отрезков

$$
B_{1} \supset W_{1} \supset B_{2} \supset W_{2} \supset \cdots
$$

с длинами $\ell\left(B_{i}\right)=2(\alpha \beta)^{i-1} \rho$ и $\ell\left(W_{i}\right)=2 \alpha(\alpha \beta)^{i-1} \rho, i=1,2, \ldots$ Очевидно, что множество $\bigcap_{i=1}^{\infty} B_{i}=\bigcap_{i=1}^{\infty} W_{i}$ состоит из одной единственной точки. Если $\bigcap_{i=1}^{\infty} B_{i} \in S$, то говорят, что белые вьиграли игру, иначе игру выиграли черные. Далее, множество $S$ назьвается $(\alpha, \beta, \rho)$-выигрышным, если белые могут выиграть игру независимо от того, как играют черные. Множество $S$ назьвается $(\alpha, \beta, \rho)$-вполне выигрышным, если для всех $\rho^{\prime} \geqslant \rho$ это множество является $\left(\alpha, \beta, \rho^{\prime}\right)$-выигрьшшным.

ЗАмЕЧАНИЕ 1 . Если множество $S$ ( $\alpha, \beta, \rho)$-вьпгрышно, то множество $a S+b$ является $(\alpha, \beta, a \rho)$-выигрышным.

ЗАмЕчАниЕ 2 . Из $(\alpha, \beta, \rho)$-выигрышности не следует $(\alpha, \beta, \rho)$-вполне вьигрышность. Так, например, множество $S=\bigcup_{n \in \mathbb{Z}}[2 n, 2 n+1)$ является $\left(1 / 3,1 / 3,3^{k} / 2\right)$-выигрышным, если $k$ нечетное натуральное, и не является $\left(1 / 3,1 / 3,3^{k} / 2\right)$-выигрышным, если $k$ четное натуральное.

Лемма 1. Пусть $0<\alpha<1,0<\beta<1, \gamma=1+\alpha \beta-2 \alpha>0, \rho>0$, u nycmb $t \in \mathbb{N}$ такое, что $(\alpha \beta)^{t}<\gamma / 2$. Пусть отрезок $B_{k}$ длины $2 \rho_{k}$ и с иентром в точке $b_{k}$ получается в $(\alpha, \beta, \rho)$ игре. Тогда белье могут играть так, что при любой игре черных отрезок $B_{k+t}$ лежит в полупрямой $x>b_{k}+\rho_{k} \gamma / 2$.

ЗАмечание 3 . Вьполнено неравенство $\gamma<1$. 
ДоКАЗАТЕЛЬСТВО ЛЕмМЫ 1. Пусть белые придерживаются такой стратегии: ходят как можно правее. Тогда

$$
\begin{gathered}
w_{k}=b_{k}+\rho_{k}(1-\alpha) \\
b_{k+1} \geqslant w_{k}-\alpha \rho_{k}(1-\beta)=b_{k}+\rho_{k}(1-\alpha-\alpha+\alpha \beta)=b_{k}+\rho_{k} \gamma>b_{k} .
\end{gathered}
$$

Отсюда видна монотонность последовательности $b_{k}$; в частности, из нее следует неравенство

$$
b_{k+t} \geqslant b_{k+1} \geqslant b_{k}+\rho_{k} \gamma
$$

Поскольку $\rho_{k+t}=\rho_{k}(\alpha \beta)^{t}<\rho_{k} \gamma / 2$, то для всех $x \in B_{k+t}$ вьполнено неравенство

$$
x \geqslant b_{k+t}-\rho_{k+t}>b_{k}+\frac{\rho_{k} \gamma}{2} .
$$

Лемма 1 доказана.

ЛЕмма 2. Пусть $0<\alpha<1,0<\beta \leqslant 1 / 2, \rho>0$ и множество $S$ является $(\alpha, \beta, \rho)$-выигрышным. Тогда мнохсество $S$ имеет хаусдорфову размерность

$$
\mathrm{HD}(S) \geqslant \frac{\ln (1 /(2 \beta))}{\ln (1 /(\alpha \beta))}
$$

ДоКАЗАТЕЛЬСТво. Положим $N=\lfloor 1 / \beta\rfloor$. Предположим, что белые используют выигрышную стратегию и что $B_{1}$ и $W_{1}$ уже заданы. Разобьем отрезок $W_{1}$ на $N$ равных частей. Черные могут играть таким образом, что отрезок $B_{2}$ будет лежать в одной из $N$ частей отрезка $W_{1}$. Обозначим $B_{2}$ через $B_{2}^{i_{1}}, i_{1} \in\{1, \ldots, N\}$. Разобьем отрезок $W_{2}$ на $N$ равных частей. За следуюший шаг черные могут сьграть так, что отрезок $B_{3}$ будет лежать в одной из $N$ частей отрезка $W_{2}$. Таким образом, существуют $N^{2}$ возможности выбора отрезка $B_{3}$, которые обозначим через $B_{3}^{i_{1} i_{2}}, i_{1}, i_{2} \in\{1, \ldots, N\}$, и т.д. Для всякой последовательности $\left(i_{k}\right)_{k=1}^{\infty}, i_{k} \in\{1, \ldots, N\}$, мы получаем последовательность отрезков $B_{2}^{i_{1}}, B_{3}^{i_{1} i_{2}}, B_{4}^{i_{1} i_{2} i_{3}}, \ldots$, пересечение которых принадлежит множеству $S$. Теперь положим

$$
A_{k}=\bigcup_{i_{1}, \ldots, i_{k} \in\{1, \ldots, N\}} B_{k+1}^{i_{1} \ldots i_{k}}
$$

Тогда $A=\bigcap_{k=1}^{\infty} A_{k} \subset S, N_{k}=N, R_{k}=N^{k}, \delta_{k}=2(\alpha \beta)^{k} \rho$. Имеет место равенство

$$
\sum_{k=2}^{\infty} \frac{\delta_{k-1}}{\delta_{k}}\left(R_{k}\left(\delta_{k}\right)^{s}\right)^{-1}=\sum_{k=2}^{\infty} \frac{1}{\alpha \beta}\left(N^{k}\left(2(\alpha \beta)^{k} \rho\right)^{s}\right)^{-1}=\frac{(2 \rho)^{-s}}{\alpha \beta} \sum_{k=2}^{\infty}\left(N(\alpha \beta)^{s}\right)^{-k} .
$$

Этот ряд сходится при $s<\ln N /|\ln (\alpha \beta)|$. Применяя теорему 3 , получим, что

$$
\mathrm{HD}(S) \geqslant \frac{\ln \lfloor 1 / \beta\rfloor}{\ln (1 /(\alpha \beta))} \geqslant \frac{\ln (1 /(2 \beta))}{\ln (1 /(\alpha \beta))} .
$$

Лемма 2 доказана.

Обозначим

$$
N_{a, b}(g)=\left\{x \in \mathbb{R} \mid \forall n \in \mathbb{N}\left\|g^{n-1}(a x+b)\right\| \geqslant g^{-1}\right\}
$$

и положим $N(g)=N_{1,0}(g)$. 
Лемма 3. Пусть $0<\alpha<1,0<\beta<1, \gamma=1+\alpha \beta-2 \alpha>0, \rho \geqslant \alpha \beta / 2$, $g \geqslant 6 /(\alpha \beta \gamma)$. Тогда множество $N(g)$ является $(\alpha, \beta, \rho)$-вполне выигрышным.

ДокАЗАТЕЛьство. Так как $\rho \geqslant \alpha \beta / 2$, то найдутся натуральные числа $n_{i}, i=0$, $1, \ldots$, такие, что

$$
\frac{4 g^{-(i+1)}}{\gamma} \leqslant g^{-i}\left(1-\frac{2}{g}\right) \alpha \beta \leqslant 2(\alpha \beta)^{n_{i}-1} \rho<g^{-i}\left(1-\frac{2}{g}\right)<1 \leqslant 2(\alpha \beta)^{-1} \rho .
$$

Так определенная последовательность натуральных чисел оказьвается строго возрастающей и, более того,

$$
\frac{(\alpha \beta)^{n_{i}}}{(\alpha \beta)^{n_{i-1}}}=\frac{2(\alpha \beta)^{n_{i}-1} \rho}{2(\alpha \beta)^{n_{i-1}-1} \rho}<\frac{g^{-i}(1-2 / g)}{g^{-(i-1)}(1-2 / g) \alpha \beta}=\frac{1}{g \alpha \beta}<\frac{\gamma}{2}<1, \quad i \in \mathbb{N} .
$$

Покажем, что белые могут играть так, что при любой игре черных

$$
\forall i \in \mathbb{N} \quad \forall x \in B_{n_{i}} \quad\left\|g^{i-1} x\right\| \geqslant g^{-1} .
$$

Пусть дан отрезок $B_{n_{i-1}}$ длины $2(\alpha \beta)^{n_{i-1}-1} \rho$ с центром в точке $b_{n_{i-1}}$. Числа $x$, для которых вьполнено неравенство $\left\|g^{i-1} x\right\| \leqslant g^{-1}$, содержатся в отрезках длины $2 g^{-i}$, расстояние между которыми

$$
g^{-(i-1)}-2 g^{-i}=g^{-(i-1)}\left(1-\frac{2}{g}\right)>2(\alpha \beta)^{n_{i-1}-1} \rho=2 \rho\left(B_{n_{i-1}}\right) .
$$

Это означает, что отрезок $B_{n_{i-1}}$ пересекается не более, чем с одним таким "плохим" отрезком $I$. Не ограничивая обшности, можно считать, что центр отрезка $I$ лежит не правее центра отрезка $B_{n_{i-1}}$. Тогда весь отрезок $I$ содержится в полупрямой

$$
\begin{aligned}
x & \leqslant b_{n_{i-1}}+g^{-i} \leqslant b_{n_{i-1}}+g^{-(i-1)}\left(1-\frac{2}{g}\right) \frac{\alpha \beta \gamma}{4} \\
& \leqslant b_{n_{i-1}}+(\alpha \beta)^{n_{i-1}-1} \rho \frac{\gamma}{2}=b_{n_{i-1}}+\rho\left(B_{n_{i-1}}\right) \frac{\gamma}{2} .
\end{aligned}
$$

Определив $t_{i}=n_{i}-n_{i-1}$, получаем

$$
(\alpha \beta)^{t_{i}}=\frac{(\alpha \beta)^{n_{i}}}{(\alpha \beta)^{n_{i-1}}}<\frac{\gamma}{2} .
$$

Теперь из леммы 1 следует, что для всех $x \in B_{n_{i}}=B_{n_{i-1}+t_{i}}$ вьполнено неравенство

$$
x>b_{n_{i-1}}+\rho\left(B_{n_{i-1}}\right) \frac{\gamma}{2} .
$$

Итак, лемма 3 доказана.

СледСтвиЕ 3. Пусть $0<\alpha<1,0<\beta<1, \gamma=1+\alpha \beta-2 \alpha>0, \rho \geqslant \alpha \beta / 2$, $g \geqslant 6 /(\alpha \beta \gamma), a \geqslant 1$. Тогда множество $N_{a, b}(g)$ является $(\alpha, \beta, \rho)$-выигрышным. 
ДокАЗАТЕЛЬСтво. Имеем

$$
\begin{aligned}
N_{a, b}(g) & =\left\{x \in \mathbb{R} \mid\left\|g^{n-1}(a x+b)\right\| \geqslant g^{-1} \forall n \in \mathbb{N}\right\} \\
& =\frac{1}{a}\left\{x \in \mathbb{R} \mid\left\|g^{n-1} x\right\| \geqslant g^{-1} \forall n \in \mathbb{N}\right\}-\frac{b}{a}=\frac{1}{a} N(g)-\frac{b}{a} .
\end{aligned}
$$

В силу леммы 3 множество $N(g) \quad(\alpha, \beta, \rho)$-вполне выигрышно. Тогда в силу замечания 1 множество $N_{a, b}(g)(\alpha, \beta, \rho / a)$-вполне вьигрьшшо. А так как $a \geqslant 1$, то множество $N_{a, b}(g)(\alpha, \beta, \rho)$-вьпгрышно.

Следствие доказано.

Пусть натуральньй ряд $\mathbb{N}$ содержит дизъюнктное объединение $\bigsqcup_{j=1}^{\infty} P_{j}$ арифметических прогрессий $P_{j}$ с первьм членом $m_{j}$ и разностью $d_{j}$. Пусть $\alpha$ и $\beta$ удовлетворяют условиям

$$
1+\alpha \beta-2 \beta>0, \quad 1-2 \alpha \geqslant 0 .
$$

Определим последовательно для всех $j \in \mathbb{N}$ следующие величины:

$$
\alpha_{j}=\alpha, \quad \beta_{j}=\beta(\alpha \beta)^{d_{j}-1}, \quad \gamma_{j}=1+\alpha_{j} \beta_{j}-2 \alpha_{j}, \quad \rho_{j}=(\alpha \beta)^{m_{j}-1} .
$$

Лемма 4. Пусть мнохсества $N_{j}\left(\alpha_{j}, \beta_{j}, \rho_{j}\right)$-выигрышны. Тогда $\bigcap_{j=1}^{\infty} N_{j}(\alpha, \beta$, 1)-выигрышно.

ДокАЗАТЕЛЬСтво. Белые играют следуюшим образом. Для ходов с номерами $m_{j}+k d_{j} \in P_{j}, k \in \mathbb{N}_{0}$, они используют $\left(\alpha, \beta(\alpha \beta)^{d_{j}-1},(\alpha \beta)^{m_{j}-1}\right)$-вьцгрьшную стратегию для множества $N_{j}$. Следовательно, $\bigcap_{k=1}^{\infty} B_{k} \in N_{j}$. Таким образом, белые могут сьграть так, чтобы $\bigcap_{k=1}^{\infty} B_{k} \in \bigcap_{j=1}^{\infty} N_{j}$.

Тем самым, лемма 4 доказана.

4. О вложении непересекающихся арифметических прогрессий в натуральный ряд. Следующее утверждение немного уточняет соответствующую лемму из работы [8].

ЛЕмма 5. Пусть $\left(v_{j}\right)_{j=1}^{\infty}-$ последовательность положительных действительных чисел таких, что $\sum_{j=1}^{\infty} v_{j}^{-1} \leqslant 1 / 2$. Тогда существует последовательность непересекаюшихся арифметических прогрессий

$P_{j}=\left\{n \in \mathbb{N} \mid n \equiv m_{j}\left(\bmod d_{j}\right)\right\}, \quad$ де $m_{j}$ u $d_{j}$-некоторые натуральные числа, maкux, чmо $v_{j} / 2<d_{j} \leqslant v_{j}$.

ЗАмечаниЕ 4. Для любого $j \in \mathbb{N}$ выполнены неравенства $v_{j} \geqslant 2$ и $m_{j} \leqslant d_{j}$.

ДоКАЗАТЕЛЬСТВо ЛЕмМЫ 5. Не ограничивая общности, можно считать, что последовательность $\left(v_{j}\right)_{j=1}^{\infty}$ монотонно возрастает. Определим последовательность натуральных чисел $\left(n_{j}\right)_{j=1}^{\infty}$ из следуюших соотношений:

$$
1 \leqslant \frac{v_{j}}{2}<2^{n_{j}} \leqslant v_{j}
$$

Далее, положим $d_{j}=2^{n_{j}}$. Заметим, что при этом $\sum_{j=1}^{\infty} d_{j}^{-1}<1$ и последовательность $\left(n_{j}\right)_{j=1}^{\infty}$ монотонно возрастает. 
Мы построим по индукции последовательность $P_{1}, P_{2}, \ldots, P_{k}, \ldots$ непересекаюшихся арифметических прогрессий с разностями соответственно $d_{1}, d_{2}, \ldots, d_{k}, \ldots$ В качестве арифметической последовательности $P_{1}$ возьмем любую арифметическую прогрессию с разностью $d_{1}$. Пусть уже построены непересекающиеся прогрессии $P_{1}, P_{2}, \ldots, P_{k}$. Так как плотность множества $\bigcup_{j=1}^{k} P_{j}$ равна $\sum_{j=1}^{k} d_{j}^{-1}<1$, то существует натуральное число $m_{k+1} \notin \bigcup_{j=1}^{k} P_{j}$. Это значит, что для любого $j \in\{1,2, \ldots, k\}$ выполнено $m_{k+1} \not \equiv m_{j}\left(\bmod 2^{n_{j}}\right)$. А это, в свою очередь, означает, что прогрессия

$$
P_{k+1}=\left\{n \in \mathbb{N} \mid n \equiv m_{k+1}\left(\bmod d_{k+1}\right)\right\}
$$

не пересекается с предыдушими прогрессиями.

Лемма 5 доказана.

\section{5. Доказательство основного результата.}

ТЕОремА 4. Пусть $\left(v_{j}\right)_{j=1}^{\infty}-$ последовательность полохсительных действительных чисел таких, что $\sum_{j=1}^{\infty} v_{j}^{-1} \leqslant 1 / 2,\left(q_{j}\right)_{j=1}^{\infty}-$ последовательность иелых чисел таких, что $q_{j} \geqslant 2,\left(a_{j}\right)_{j=1}^{\infty}-$ последовательность действительных чисел таких, что $a_{j} \geqslant 1,\left(b_{j}\right)_{j=1}^{\infty}-$ произвольная последовательность действительных чисел, $\varkappa \geqslant \varkappa_{0}=\ln \left(2^{18} 3^{2}\right)=14.67387383 \ldots$ Тогда существует множсество $P_{\varkappa}$ вешественных чисел $x$ такое, что

$\forall x \in P_{\varkappa} \quad \forall n \in \mathbb{N}_{0} \quad \forall j \in \mathbb{N} \quad$ выполнена оченка $\left\|q_{j}^{n}\left(a_{j} x+b_{j}\right)\right\| \geqslant \exp \left(-\varkappa v_{j}\right)$ $u \operatorname{HD}\left(P_{\varkappa}\right) \geqslant 1-\varkappa_{0} / \varkappa$.

ЗАМЕЧАнИЕ 5 . Если $\varkappa \rightarrow \infty$, то $\mathrm{HD}\left(P_{\varkappa}\right) \rightarrow 1$.

Теоремы 1 и 2 являются следствиями теоремы 4 . Теорема 1 получается, если в условии теоремы 4 положить

$$
q_{j}=2, \quad a_{j}=3^{j}, \quad b_{j}=0 \quad \forall j \in \mathbb{N} .
$$

Теорема 2 получается, если в условии теоремы 4 положить

$$
q_{j}=j+1, \quad a_{j}=1, \quad b_{j}=0 \quad \forall j \in \mathbb{N} .
$$

ДокАЗАТЕЛЬСТво ТЕОРЕМЫ 4. Пусть $\tau=2 \varkappa / \varkappa_{0}, \alpha=1 / 2, \beta=(1 / 2)^{\tau-1}, m_{j}, d_{j}-$ числа из леммы 5 и $\alpha_{j}, \beta_{j}, \rho_{j}$ выбраны согласно (3). Определим $s_{j} \in \mathbb{N}$ и $g_{j}$ из соотношений

$$
\begin{gathered}
\frac{6}{\alpha_{j} \beta_{j} \gamma_{j}} \leqslant q_{j}^{s_{j}}<\left(\frac{6}{\alpha_{j} \beta_{j} \gamma_{j}}\right)^{2}, \quad g_{j}=q_{j}^{s_{j}}, \quad \text { если } q_{j}<\frac{6}{\alpha_{j} \beta_{j} \gamma_{j}}, \\
\frac{6}{\alpha_{j} \beta_{j} \gamma_{j}} \leqslant q_{j}^{1 / s_{j}}<\left(\frac{6}{\alpha_{j} \beta_{j} \gamma_{j}}\right)^{2}, \quad g_{j}=q_{j}^{1 / s_{j}}, \quad \text { если } q_{j} \geqslant \frac{6}{\alpha_{j} \beta_{j} \gamma_{j}} .
\end{gathered}
$$

Оценим снизу хаусдорфову размерность множества точек $x \in \mathbb{R}$, удовлетворяющих для любых $n \in \mathbb{N}_{0}, j \in \mathbb{N}$ неравенству

$$
\left\|q_{j}^{n}\left(a_{j} x+b_{j}\right)\right\| \geqslant\left(\frac{\alpha_{j} \beta_{j} \gamma_{j}}{6}\right)^{4} .
$$


Из соотношений (4) и замечания к лемме 5 следует, что вьполняются условия следствия леммы 3 и, значит, множество $N_{a_{j}, b_{j}}\left(g_{j}\right)$ является $\left(\alpha_{j}, \beta_{j}, \rho_{j}\right)$-выпгрышным. Из соотношений (3) и леммы 4 следует, что пересечение $P$ всех множеств $N_{a_{j}, b_{j}}\left(g_{j}\right)$ является $(\alpha, \beta, 1)$-вьигрышньп. А из соотношений $(2)$ и леммы 2 следует, что это множество имеет хаус дорфову размерность

$$
\mathrm{HD}(P) \geqslant \frac{\ln (1 /(2 \beta))}{\ln (1 /(\alpha \beta))}=1-\frac{\varkappa_{0}}{\varkappa}
$$

Кроме того, оно состоит из тех $x \in \mathbb{R}$, для которых для любого $n \in \mathbb{N}_{0}$ и любого $\forall j \in \mathbb{N}$ выполнено

$$
\left\|g_{j}^{n}\left(a_{j} x+b_{j}\right)\right\| \geqslant g_{j}^{-1}
$$

Выражая $g_{j}$ через $q_{j}$, эту оценку можно записать так:

$$
\begin{aligned}
\left\|q_{j}^{s_{j} n}\left(a_{j} x+b_{j}\right)\right\| \geqslant q_{j}^{-s_{j}} \quad \forall n \in \mathbb{N}_{0}, \quad \text { если } q_{j}<\frac{6}{\alpha_{j} \beta_{j} \gamma_{j}}, & \\
\left\|q_{j}^{n / s_{j}}\left(a_{j} x+b_{j}\right)\right\| \geqslant q_{j}^{-1 / s_{j}} \quad \forall n \in \mathbb{N}_{0}, & \text { если } q_{j} \geqslant \frac{6}{\alpha_{j} \beta_{j} \gamma_{j}},
\end{aligned}
$$

Пусть $q_{j}<6 /\left(\alpha_{j} \beta_{j} \gamma_{j}\right)$; тогда в $q_{j}$-ичной записи числа $a_{j} x+b_{j}$ не может стоять $2 s_{j}$ нулей подряд и $2 s_{j}$ цифр " $q_{j}-1$ ” подряд, т.е.

$$
\left\|q_{j}^{n}\left(a_{j} x+b_{j}\right)\right\| \geqslant q_{j}^{-2 s_{j}} \quad \forall n \in \mathbb{N}_{0}
$$

Применяя неравенство (4), получим неравенство (5).

Пусть $q_{j} \geqslant 6 /\left(\alpha_{j} \beta_{j} \gamma_{j}\right)$; тогда, подставляя в (6) $n=k s_{j}, k \in \mathbb{N}_{0}$, получим

$$
\left\|q_{j}^{k}\left(a_{j} x+b_{j}\right)\right\| \geqslant q_{j}^{-1 / s_{j}} \quad \forall k \in \mathbb{N}_{0}
$$

Применяя неравенство (4), получим неравенство (5).

Учитывая определения величин $\alpha_{j}, \beta_{j}, \gamma_{j}$ (соотношения (3)), неравенство (5) и неравенства леммы 5 , получаем для любого $n \in \mathbb{N}_{0}$ и любого $\forall j \in \mathbb{N}$

$$
\left\|q_{j}^{n}\left(a_{j} x+b_{j}\right)\right\| \geqslant\left(\frac{\alpha_{j} \beta_{j} \gamma_{j}}{6}\right)^{4}=\frac{1}{6^{4}} \cdot\left(\frac{1}{2}\right)^{8 d_{j} \tau} \geqslant \exp \left(-\varkappa v_{j}\right) .
$$

Теорема 4 доказана.

ЗАмЕчАнИЕ 6 . В доказательстве теоремы при $q_{j} \leqslant 6 /\left(\alpha_{j} \beta_{j} \gamma_{j}\right)$ можно сделать оценку лучше, но это, по-видимому, не представляет интереса.

Автор глубоко признателен рецензенту за ряд существенных замечаний к рукописи, позволивших несколько улучшить основной результат. 


\section{СПИСОК ЦИТИРОВАННОЙ ЛИТЕРАТУРЫ}

[1] Erdös P. Problems and results on Diophantine approximations. II // Répartition modulo 1 (Actes Colloq., Marseille-Luminy, 1974). Lecture Notes in Math. V. 475. Berlin: Springer-Verlag, 1975. P. 89-99.

[2] de Mathan D. Numbers contravening a condition in density modulo 1 // Acta Math. Acad. Sci. Hungar. 1980. V. 36. №3-4. P. 237-241.

[3] Pollington A.D. On the density of sequence $\left\{n_{k} \zeta\right\}$ // Illinois J. Math. 1979. V. 23. P. 511-702.

[4] Boshernitzan M. Density modulo 1 of dilations of sublacunary sequences // Adv. in Math. 1994. V. 108. P. 104-117.

[5] Furstenberg H. Disjointness in ergodic theory, minimal sets, and a problem in diophantine approximation // Math. Systems Theory. 1967. V. 1. P. 1-49.

[6] Boshernitzan M.D. Elementary proof of Furstenberg's diophantine result // Proc. Amer. Math. Soc. 1994. V. 122. №1. P. 67-70.

[7] Schmidt W. M. On badly approximable numbers and certain games // Trans. Amer. Math. Soc. 1966. V. 123. №1. P. 67-70.

[8] Ахунжанов Р. К. Об анормальных числах // Матем. заметки. 2002. Т. 72. № 1. С. 150-152.

[9] Eggleston H. G. Sets of fractional dimension which occur in some problems of number theory // Proc. London Math. Soc. 1951-52. V. 54. P. 42-93.

[10] Шмидт В. М. Диофантовы приближения. М.: Мир, 1983.

Московский государственный университет им. М.В. Ломоносова

Поступило

18.09.2003

Исправленный вариант

29.12 .2003 